Guest Editorial

\title{
A Strategic Roadmap for Computing for Science and Engineering
}

\author{
R K SHYAMASUNDAR \\ Department of Computer Science and Engineering, Indian Institute of Technology Bombay, Mumbai, \\ India
}

Year 2011 was the centenary year of Homi Bhabha - the founder of Tata Institute of Fundamental Research (TIFR) and other key Science \& Technology establishments in India. To commemorate the centenary, I had organized a conference on Computing for Science Discovery and Innovations: A Roadmap at TIFR, Mumbai. During the conference, some of my friends and colleagues persuaded me to take up writing of a report on Computing for Science, Engineering, and Society: Challenges, Requirement, and Strategic Roadmap, under the solicited programme of "Commissioning of well-researched in-depth reports on topics of scientific and societal importance" from the Indian National Science Academy. It was certainly a good idea but I underestimated the challenges involved in undertaking the task. It is indeed gratifying that many of my friends and colleagues rescued me by identifying several authors who could potentially contribute to the task, as it was not the task of a single person. I am indeed extremely grateful to my co-authors who have contributed in bringing out various challenges by discussing the basis of prioritization in their sub-areas. The full report is available here. This Special Section on "Computing for Science, Engineering, and Society: Challenges, Requirement, and Strategic Roadmap" includes the Executive Summary and the Chapters 2, 3, 4, 8 and 10 of the full report since these provide essential details of what we believe are doables.

Providing a comprehensive roadmap is almost an impossible task due to the dynamic and the ubiquitous nature of computing and communication. In this report, we shall highlight some of the areas like HPC challenges for science, engineering and society, big data applications, HPC requirements and impact on public infrastructures and glimpses to some of the possibly disruptive research areas of computer science including AI, cyber security, MOOCs, and computational thinking. Each chapter is structured to bring out a main challenge and a few takeaways to meet the challenge or get the best for the society from the investment in the sector. The report is organized as follows:

- In Chapter 2, RK Shyamasundar provides a brief reflection on evolution and future of computer science; a brief account of some of the research challenges of AI, cyber security, MOOCs are also highlighted.

- In Chapter 3, Inder Monga and Prabhat explore the impact on Information and Computing Technology (ICT) infrastructure on science and highlight their experiences from the Energy Sciences Network of USA.

- In Chapters 4 and 5, Ashwin Gumaste brings out the requirements of network computing, challenges and the impact it can generate in Indian context from his experiences of building SDN routers at IIT Bombay and deployed across India. It also provides a lead for one to think as to how the local innovations in frontier technologies need a support base from the industry, business, and the Government to take it forward.

- Vipin Chaudhary explores the role of Big Data in Government in Chapter 6, in which he brings out his research experiences that include his findings from the role Big Data is playing in USA. 
- Ankur Narang who was with IBM India Research Laboratory while writing the report explores HPC applications in smart-grid, oil and natural gas in Chapter 7.

- In Chapter 8, RK Shyamasundar and Vishwas $\mathrm{T}$ Patil explore the supposedly disruptive blockchain technology in finance and governance.

- In Chapter 9, RK Shyamasundar briefly highlights Computational Thinking — a paradigm that is building bridges across various science disciplines through the computing paradigm.

- $\quad$ The report concludes in Chapter 10 with a very broad takeaways that policy-makers can have on "the impact of computing paradigm on science, and society."

- The report also has an Appendix that highlights the recommendations of various experts in the context of a conference held in connection the centenary celebration of Homi Bhabha at TIFR.

There has been a delay in arriving at the report owing to a spectrum of challenges including my movement from TIFR to IIT Bombay in early 2015. In spite of the delay, I hope that this report will be useful to a large section of the communities involved in R\&D activities relying on computing infrastructure.

Having got the manuscripts in various formats from the co-authors, to place the reports in an uniform book format was really a gigantic task. Vishwas Patil (CSE, IIT Bombay) took the task and typeset it using LaTeX. I am indeed grateful to him. Initial reading, suggestions and proof reading were done by NV Narendra Kumar (TIFR/IIT Bombay, currently with IDRBT Hyderabad) and Vishwas Patil. I am extremely thankful to both of them for their suggestions and careful reading of the manuscripts. It is indeed a pleasure to thank STCS at TIFR and Department of CSE at IIT Bombay for all the facilities provided while writing this report.

\section{Current Affiliations of Authors}

Professor Vipin Chaudhary

Department of Computer Science

SUNY Buffalo

(Former CEO, Computational Research Lab. (CRL), Tata Sons. Ltd.);

Currently duty at NSF and also on HPC advisory board of the US Council of Competitiveness

vipin@buffalo.edu

Professor Ashwin Gumaste

Department of Computer Science and Engineering

IIT Bombay

ashwin@cse.iitb.ac.in

Dr. Inder Monga

Lawrence Berkeley National Lab

University of Berkeley

imonga@es.net

Dr. Ankur Narang

Associate Vice President - Data Science, Chief

Data Scientist at Mobileum

(Formerly with IBM India Research Labs)

annarang2@gmail.com

Dr. Vishwas T Patil

Department of Computer Science and Engineering

IIT Bombay

ivishwas@gmail.com

Dr. Prabhat

National Energy Research Scientific Computing

Center, USA

prabhat@lbl.gov

Professor R K Shyamasundar

Department of Computer Science and Engineering

IIT Bombay

rkss@cse.iitb.ac.in 